\title{
A Novel Gene RH4, Inhibiting Pigment Deposition in Rice Hull Furrows by Participating in Flavonoid Biosynthesis
}

\author{
${ }^{1}$ Meijuan Duan , ${ }^{2}$ Ling Liu \\ ${ }^{1}$ Hunan Agricultural University \\ No.1, Nongda Road, Furong District, Changsha, China \\ duanmeijuan@163.com; liuling_66@hnu.edu.cn \\ ${ }^{2}$ Long Ping Branch, Graduate School of Hunan University, Changsha \\ No. 844, Yuanda Er Road, Furong District, Changsha, China
}

\section{Extended Abstract}

Rice (Oryza sativa) specific color hull phenotype is a classical morphological marker that has long been applied to breeding and genetics study. The discovery and utilization of specific genetic resources provided a new strategy for innovative seed production technology. Recently, several mutants which had been reported showed abnormal hull colors, brown or black, e.g. $g h 1, g h 2, g h 3, g h 4, g h 5, g h 6, b h 4, b h 6, i b f 1, c a d 2$ and $l s i 1[1-6]$, and the function of these corresponding genes were usually involved in flavonoid biosynthesis. However, less is known about the mechanism of flavonoid biosynthesis and metabolism regulating in rice. In this study, we characterized a natural mutant with red pigmentation in the hull furrows in the background of cultivated rice variety $O$. sativa indica $c v$ Xianhui207 based on forward genetic method, which was termed as $r h 4$. Compared with other marker traits, the stable red hull phenotype of rh4 mutant is more powerful and intuitive for the rapid selection of hybrid seeds to solve the current critical technical problems in mixed-sowing seed production. $\mathrm{RH} 4$ gene was cloned in rice via a map-based cloning approach. RH4 encodes an uncharacterized protein and contains a transmembrane domain, which is similar to a generally expressed protein. RH4 expresses in most tissues of rice and most abundantly in hulls. RH4 was localized not only on the nucleus but also at the plasma membrane, which suggests RH4 may play an important part in activating or suppressing the expression of downstream genes in flavonoid biosynthesis and may be taken as a signal conduction receptor. The mutation of $R H 4$ caused that the relative expression level of some key genes related to the flavonoid biosynthesis including $\mathrm{CHS}$ and $\mathrm{CHI}$ could be up- or down- regulated to some different extent in $r h 4$ mutant via real-time PCR, which also verified by the proteomic analysis. The Whole Genome Bisulfite-seq (WGBS) analysis displayed there were several hypo differentially methylated regions (DMRs) genes e.g. F3' $\mathrm{H}, \mathrm{F} 3$ '5' $\mathrm{H}$ in $\mathrm{CHH}$ in flavonoid biosynthesis pathway. We also detected three remarkable phosphoproteins in flavonoid biosynthesis in $r h 4$ mutant, such as CHS (134T, 1.427), CHI (232S, 179S, 177S, 2.006) and F3'H (178S, 275S, 107S, 1.429). These data implied RH4 may regulate flavonoids biosynthesis from epigenetic modification and post-translational levels. Moreover, profiles of several sorts of flavonoids was changed significantly, e.g. Cyanidin 3-[6-(3-glucosylcaffeyl) glucoside]-5-glucoside. Some anthocyanin content were reduced compared to wild type, e,g. prunin 6"-O-gallate was distinctly decreased fourfold, while the procyanidins were elevated, and catechin 7-O-apiofuranoside which showed red pigmentation was increased twofold. These findings demonstrated $R H 4$ indirectly took part in the flavonoid biosynthesis pathway in rice and made flavonoids flux from procyanidins to anthocyanin. Our results suggest RH4 may inhibit reddish-brown pigmentation in the hull furrows from participating inflavonoid biosynthesis.

\section{References}

[1] K. W. Zhang, Q. Qian, Z. J. Huang, Y. Q. Wang, M. Li, L. L. Hong, D. L. Zeng, M. H. Gu, C. C. Chu, Z. K. Cheng, "GOLD HULL AND INTERNODE2 encodes a primarily multifunctional cinnamyl-alcohol dehydrogenase in rice1," Plant Physiol., vol. 140, no. 3, pp. 972-983, 2006.

[2] B. F. Zhu, L. Z. Si, Z. X. Wang, Y. Zhou, J. J. Zhu, Y. Y. Shangguan, D. F. Lu, D. L. Fan, C. Y. Li, H. X. Lin, Q. A. Qian, T. Sang, B. Zhou, Y. Z. Minobe, B. Han, "Genetic control of a transition from black to straw-white seed hull in rice domestication," Plant Physiol. vol. 155, no. 3, pp. 1301-1311, 2011.

[3] L. L. Hong, Q. Qian, D. Tang, K. J. Wang, M. Li, Z. K. Cheng, "A mutation in the rice chalcone isomerase gene causes the golden hull and internode 1 phenotype," vol. 236, no. 1, pp. 141-151, 2012. 
[4] T. Shao, Q. Qian, D. Tang, J. Chen, M. Li, Z. K. Cheng, Q. Luo, "A novel gene IBF1 is required for the inhibition of brown pigment deposition in rice hull furrows," Theor Appl Genet., vol. 125, no. 2, pp. 381- 390, 2012.

[5] T. Koshiba, S. Murakami, T. Hattori, M. Mukai, A. Takahashi, A. Miyao, H. Hirochika, S. Suzuki, M. Sakamoto, T. Umezawa, "CAD2 deficiency causes both brown midrib and gold hull and internode phenotypes in Oryza sativa L. cv.," Nipponbare Plant Biotechnol-Nar., vol. 30, no. 4, pp. 365-373, 2013.

[6] Chengcong Yang, Dongdong Zeng, Ran Qin, Md. Alamin, Xiaoli Jin, Chunhai Shi, "Rice gene, BBH/Lsil, regulates the color of rice hull by reducing the absorption and deposition of silicon and accumulating excess flavonoid," Plant Growth Regulation., vol. 85, no.1, pp. 95-108, 2018. 